\title{
Archéologie du massif du Barajas. Premières données sur l'évolution des sociétés préhispaniques du sud-ouest du Guanajuato, Mexique
}

Grégory Pereira, Gérald Migeon et Dominique Michelet

\section{OpenEdition}

\section{Journals}

Édition électronique

URL : http://journals.openedition.org/jsa/1893

DOI : $10.4000 /$ jsa. 1893

ISSN : 1957-7842

Éditeur

Société des américanistes

Édition imprimée

Date de publication : 1 janvier 2001

Pagination : 265-281

ISSN : 0037-9174

Référence électronique

Grégory Pereira, Gérald Migeon et Dominique Michelet, «Archéologie du massif du Barajas. Premières données sur l'évolution des sociétés préhispaniques du sud-ouest du Guanajuato, Mexique », Journal de la société des américanistes [En ligne], 87 | 2001, mis en ligne le 29 juillet 2006, consulté le 19 avril 2019. URL : http://journals.openedition.org/jsa/1893 ; DOI : 10.4000/jsa.1893 
NOTES DE RECHERCHE

ARCHÉOLOGIE DU MASSIF DU BARAJAS

PREMIÈRES DONNÉES

SUR L'ÉVOLUTION DES SOCIÉTÉS PRÉHISPANIQUES

DU SUD-OUEST DU GUANAJUATO, MEXIQUE

\author{
Grégory PEREIRA*, Gérald MIGEON*, Dominique MICHELET*
}

Le massif du Barajas se situe à la limite sud-ouest de l'État mexicain du Guanajuato (municipe de Pénjamo). Depuis 1998, les sites qu'il abrite font l'objet de travaux archéologiques qui constituent un des volets du programme « Dynamiques culturelles dans le Bajio » ${ }^{1}$. Les questions qui animent cette recherche sont étroitement liées à un problème récurrent de l'archéologie des confins septentrionaux de la Mésoamérique : celui des fluctuations subies par la limite nord de cette aire culturelle (Braniff 1989, 1994). En effet, si l'existence de telles fluctuations est bien établie depuis les travaux pionniers de Pedro Armillas $(1964,1969)$, leurs causes et leurs modalités restent encore très largement méconnues. Cette problématique a constitué un des axes traditionnels de la recherche archéologique française au Mexique et les travaux engagés dans le sud-ouest du Guanajuato ont été précédés par d'importantes contributions portant sur divers secteurs des marges septentrionales de la Mésoamérique (StresserPéan 1977 ; Michelet 1984 ; Rodriguez Loubet 1985 ; Taladoire \& Rodriguez Loubet 1979 ; Faugère-Kalfon 1996).

Situé dans l'extrême ouest du Bajio ${ }^{2}$, sur la rive nord du fleuve Lerma qui, au $\mathrm{XVI}^{\mathrm{e}}$ siècle, matérialisait la limite culturelle entre agriculteurs sédentaires mésoméricains, au sud, et chasseurs-collecteurs nomades, au nord, le massif du Barajas occupe une position clef à l'égard de cette problématique (Figure 1). Outre le caractère stratégique de sa situation géographique, d'autres raisons ont motivé le choix de cette zone. Ces dernières tiennent essentiellement aux spécificités du principal établissement préhispanique du massif : le site de Nogales. Celui-ci avait été repéré à l'occasion de travaux de reconnaissance menés par une équipe mexicaine, au début des années quatre-vingt, en préalable à la construction du gazoduc Salamanca-Degollado et il

*Archéologie des Amériques (UMR 8096 CNRS-université Paris I), Maison de l'archéologie et de l'ethnologie, université Paris-X-Nanterre, 21, allée de l'Université, 92023 Nanterre cedex.

Journal de la Société des Américanistes 2001, 87 : p. 265 à 281. Copyright C Société des Américanistes. 


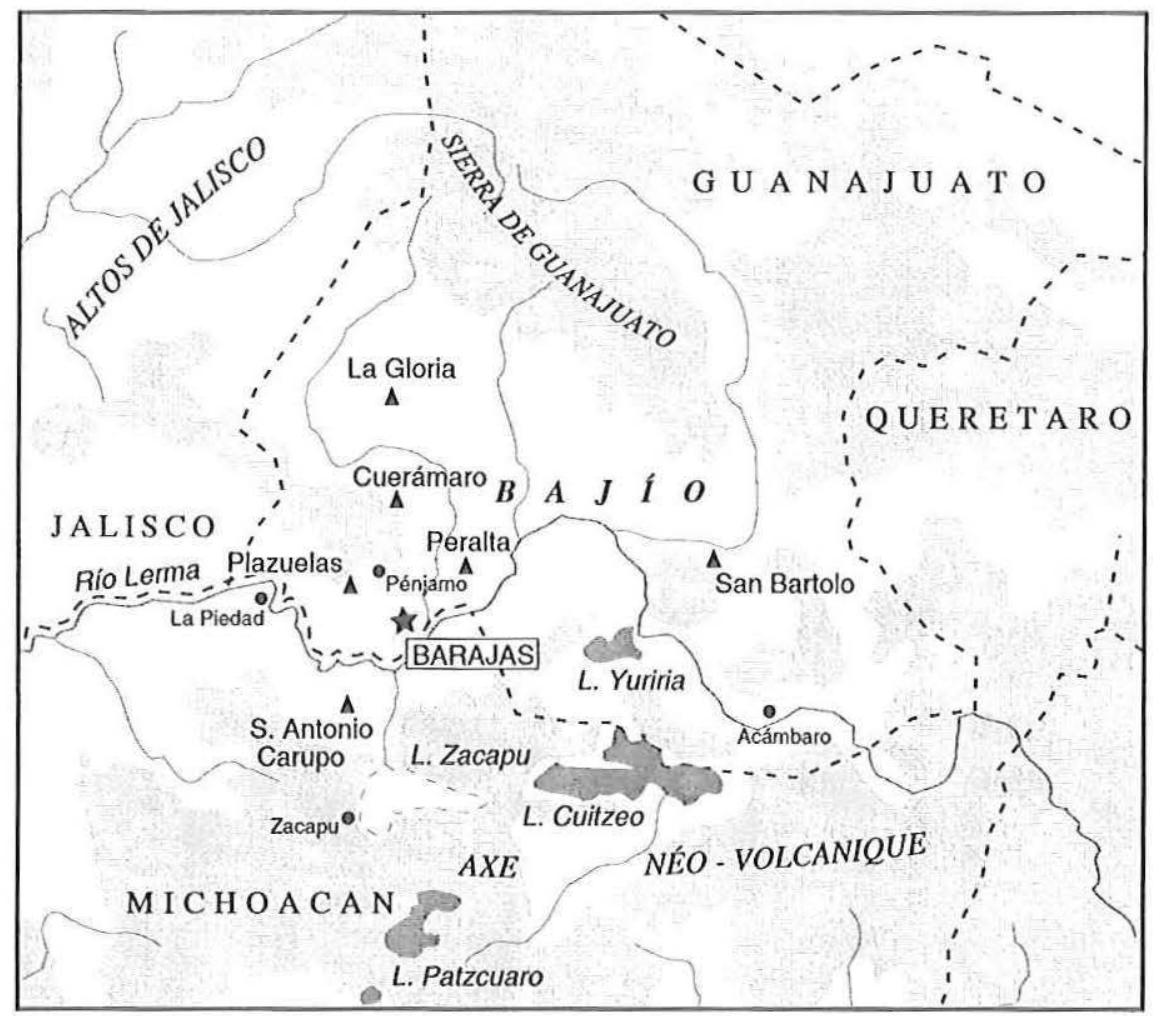

FIG. 1 - Localisation du massif du Barajas et de quelques sites importants du Bajio

avait retenu l'attention par la monumentalité de ses structures en pierre sèche. Ses inventeurs l'avaient alors interprété comme une forteresse implantée aux frontières nord du royaume protohistorique tarasque (Zepeda 1988 ; Sánchez Correa 1993). Les recherches réalisées ultérieurement par notre équipe dans le centre-nord du Michoacan (de 1983 à 1997) nous conduisaient à douter de cette attribution chrono-culturelle. Les caractéristiques architecturales de Nogales permettaient plutôt de supposer une occupation correspondant à l'Épiclassique et au début du Postclassique (700-1000 apr. J.-C.), période au cours de laquelle une grande partie des régions septentrionales est progressivement désertée par les populations mésoaméricaines tandis que divers éléments «nordiques » font leur apparition dans l'architecture de la région. À cet égard, nous supposions que Nogales pouvait s'apparenter au site de San Antonio Carupo (Michoacan) dans lequel au moins un édifice présente des ressemblances étonnantes avec des bâtiments de la culture Chalchihuites (Faugère-Kalfon 1991, 1996). Cette dernière constatation soulevait en fait le problème de l'identité des occupants du massif du Barajas et, de façon plus générale, celui des phénomènes migratoires attestés par les sources ethnohistoriques.

Par ailleurs, le bon état de conservation des sites du massif et leur localisation à proximité de secteurs étudiés dans le cadre de projets français et mexicains antérieurs 


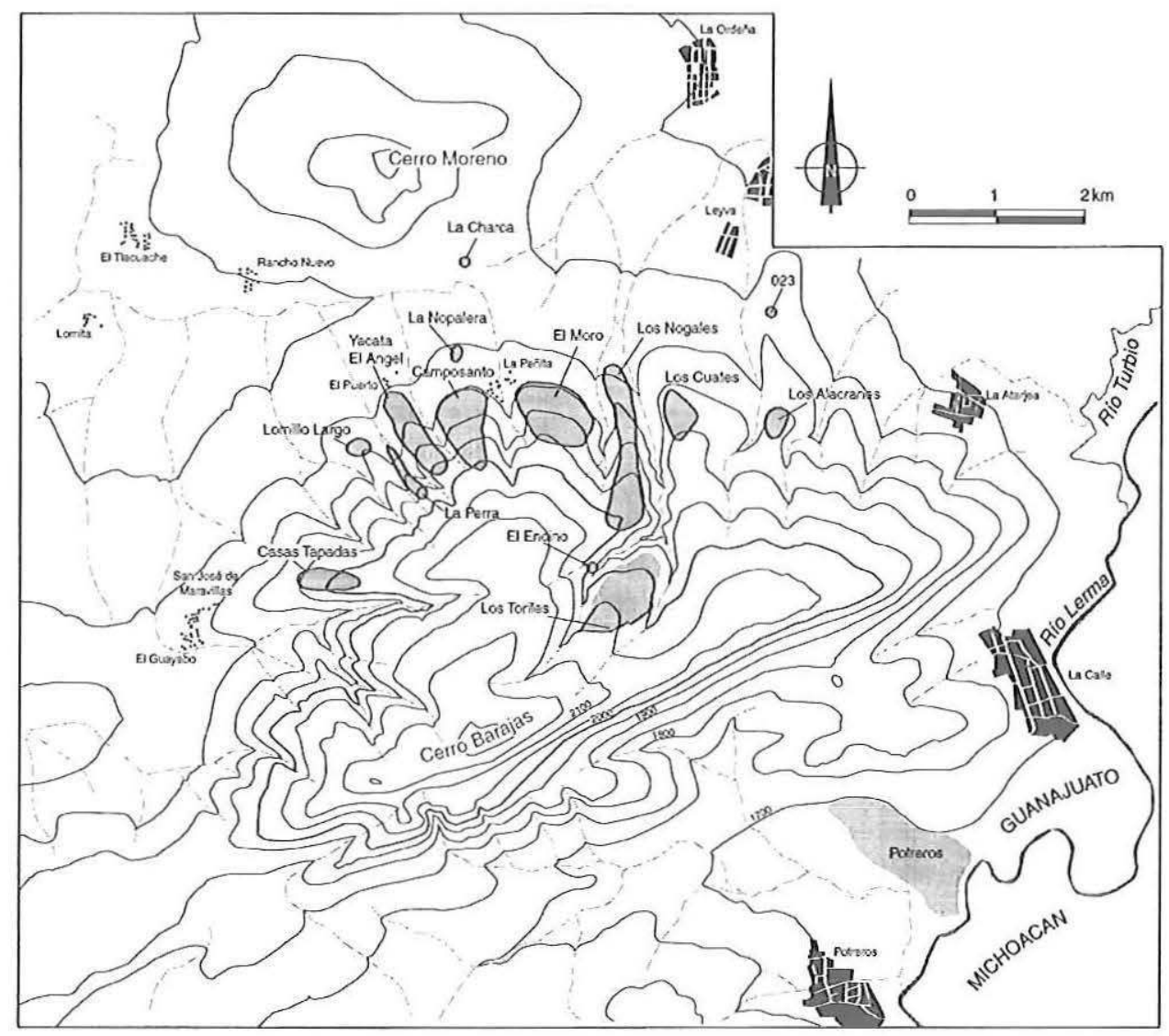

FIG. 2 - Distribution des sites sur le flanc nord du Cerro Barajas (carte G. Pereira)

ou en cours offraient des perspectives comparatives non négligeables, dans une région où l'archéologie est encore très faiblement développée ${ }^{3}$.

\section{OBJECTIFS ET TRAVAUX}

Depuis le début du projet, trois campagnes successives $(1998,1999,2000)$ comportant des opérations de prospection, topographie et fouille ont pu être réalisées. Ces travaux visaient plusieurs objectifs.

Il était tout d'abord nécessaire d'obtenir des données précises concernant l'occupation préhispanique de la zone afin de replacer le site de Nogales dans son contexte local. C'est dans cette optique qu'une prospection systématique du versant nord du massif du Barajas a été mise en œuvre afin de compléter quantitativement et qualitativement le répertoire des sites de l'Atlas archéologique dressé par l'INAH. Pour chaque établissement, une description détaillée a été effectuée, complétée par des relevés lorsque la conservation des structures le permettait et a été accompagnée de ramassages de surface. Cette opération a permis d'identifier un total de 15 sites localisés sur les versants et en contrebas du massif (Figure 2). À ce jour, les plans de 
29 groupes de structures ont été dressés sous forme de plans topographiques (22) ou de croquis à la boussole et au décamètre (7). Ces relevés constituent une base de données importante pour étudier la structuration des établissements préhispaniques (près de 140 structures et aménagements divers ont ainsi été répertoriés).

Par ailleurs, la question chronologique constituait une priorité, dans la mesure où nous ne disposions d'aucune référence solide concernant l'époque d'occupation des sites. Le matériel employé à cet effet provient d'une vingtaine de sondages et de 6 fouilles extensives effectuées dans 13 groupes de structures localisés à Nogales comme dans les sites voisins. Signalons d'emblée l'importance des fouilles menées dans le Groupe A de Nogales (Figure 3) où une stratigraphie complexe de près de $2 \mathrm{~m}$ d'épaisseur a été mise au jour lors de la fouille des structures A1, A2 et A3. À cet endroit, la mise en évidence de cinq niveaux d'occupations successifs associés à un matériel céramique relativement abondant et à des charbons a permis de construire une séquence chronologique que nous présenterons plus en détail ci-dessous.

À travers la réalisation de dégagements plus ou moins extensifs, nous avons entrepris de déterminer la fonction de plusieurs structures repérées en surface. Ces opérations ont concerné des structures résidentielles telles que les structures A1 et A2 du Groupe A de Nogales qui ont été entièrement dégagées ainsi qu'une partie du Groupe C du site de Yácata El Angel (patio et pièces associées). D'autres éléments sans doute liés au stockage ont aussi été fouillés : une concentration de petites fosses aux parois construites à l'aide de dallettes a été dégagée de façon extensive dans le Groupe D du site d'El Moro ; dans le Groupe A de Casas Tapadas, deux petites pièces localisées en sous-sol d'un bâtiment sans doute résidentiel ont également été fouillées. Enfin, certaines interventions ont porté sur des constructions non domestiques: dans le Groupe B de Nogales, nous avons dégagé le petit autel (B4) qui se trouve au centre d'une place cérémonielle bordée de trois imposantes structures pyramidales ; sur le site défensif de Los Toriles, l'entrée de la muraille a été partiellement mise au jour.

L'étude des pratiques funéraires et des caractéristiques biologiques des populations du massif constituait également un des objectifs de départ du projet. Mais ce volet du programme n'a pu être entamé que lors de la campagne 2000 avec la réalisation d'une série de sondages destinés à localiser d'éventuelles zones funéraires et à en évaluer les potentialités. Un sondage effectué dans la structure G9 du Groupe G de Nogales ouvre des perspectives intéressantes à ce sujet. Il s'agit d'une petite terrasse artificielle qui supporte les vestiges très altérés d'une construction en dalles. Le remblai de la terrasse conserve une grande densité de sépultures associées à du matériel de phase Barajas : dans les $4 \mathrm{~m}^{2}$ fouillés à ce jour; les restes plus ou moins complets de cinq dépôts primaires ont été découverts. La sépulture la mieux préservée (Sép. 3) conservait les restes d'un adulte âgé de sexe féminin déposé dans une fosse en position de décubitus dorsal avec les membres inférieurs en extension. Cette femme avait été inhumée avec les ossements d'un autre adulte (dépôt secondaire probablement) ainsi qu'un riche mobilier composé de dix récipients de céramique, d'une parure formée de petites perles de coquillage retrouvées au niveau de la taille et d'un tesson découpé en forme de jeton. Au regard de ces résultats prometteurs, il sera nécessaire d'étendre la fouille de cette zone afin d'en réaliser l'exploration systématique. 


\section{Premiers résultats}

\section{La chronologie céramique et la séquence d'occupation}

L'analyse de plus de 20000 tessons issus des différentes opérations de fouille et associés à plusieurs dates radiocarbone ${ }^{4}$ a permis de proposer une séquence céramique qui témoigne d'une occupation continue du massif depuis environ 450 de notre ère jusqu'en 1000-1100. Au sein de cet intervalle, nous avons pu discerner quatre phases d'occupation distinctes auxquelles il convient d'ajouter une cinquième phase correspondant à une réoccupation sporadique plus tardive (Xve-XvIe siècles). Ces cinq phases sont brièvement présentées ci-dessous. Précisons que pour les deux phases anciennes, les informations disponibles proviennent essentiellement des fouilles du Groupe A de Nogales, tandis que la cinquième phase n'a été identifiée, pour l'instant, que dans les couches superficielles du Groupe B du même site (voir Figure 3).

\section{Occupation initiale (autour de 450 apr. J.-C.)}

Celle-ci correspond aux couches les plus profondes mises au jour lors des fouilles de la structure A2. Étant donnée la surface limitée de la fouille dans ces niveaux, il est difficile d'établir sa nature réelle. Les vestiges disponibles montrent en tout cas que ces premiers occupants avaient commencé à modifier la topographie naturelle du lieu par la construction d'une plate-forme (ou grande terrasse) qui servit de base aux constructions ultérieures. Cette occupation paraît par ailleurs posséder une dimension cultuelle : c'est ce que suggère la présence de deux sépultures d'enfants ainsi qu'un matériel céramique de qualité avec plusieurs tessons rares, correspondant sans doute à des récipients importés (céramique stuquée, pseudo-cloisonnée, trichrome). Bien entendu, il ne faut pas exclure pour autant la possibilité d'une occupation domestique. En tout état de cause, l'occupation ancienne semble liée à la présence de plusieurs sources permanentes localisées à proximité du groupe. À l'exception des types rares mentionnés ci-dessus, la céramique associée à cette occupation présente peu de différences avec celle qui caractérise la phase suivante. On y trouve déjà bon nombre de types tels que le Blanco levantado, le Rouge sur bai ou les récipients bruns fins incisés qui sont caractéristiques des traditions céramiques du Bajio au Classique (cf. Castañeda \& al. 1988 ; Braniff 1999). Il est vraisemblable que ces premiers occupants proviennent de sites localisés dans la plaine alluviale. Quelques-uns de ces établissements ont été identifiés au pied du massif. Signalons d'ailleurs que le site de Potreros, localisé au sud-est de la zone prospectée, sur la rive du Lerma, a livré quelques indices d'une possible occupation plus ancienne.

\section{La phase Nogales (de 450/500 à 750 apr. J.-C.)}

Dans le Groupe A de Nogales, cette phase se caractérise par un épisode de construction important. Les vestiges de divers édifices datés de cette phase ont été partiellement dégagés lors des fouilles réalisées sous les structures A1, A2 et A3. L'architecture de cette époque emploie des dalles d'andésite dont les affleurements naturels abondent sur le massif. Les murs de pierre sèche sont épais et les parements sont formés par la tranche brute des dalles qui ne montre pas de traces de taille. Les sols sont faits d'une chape de terre blanchâtre ou jaune orangé formée de roche altérée. 


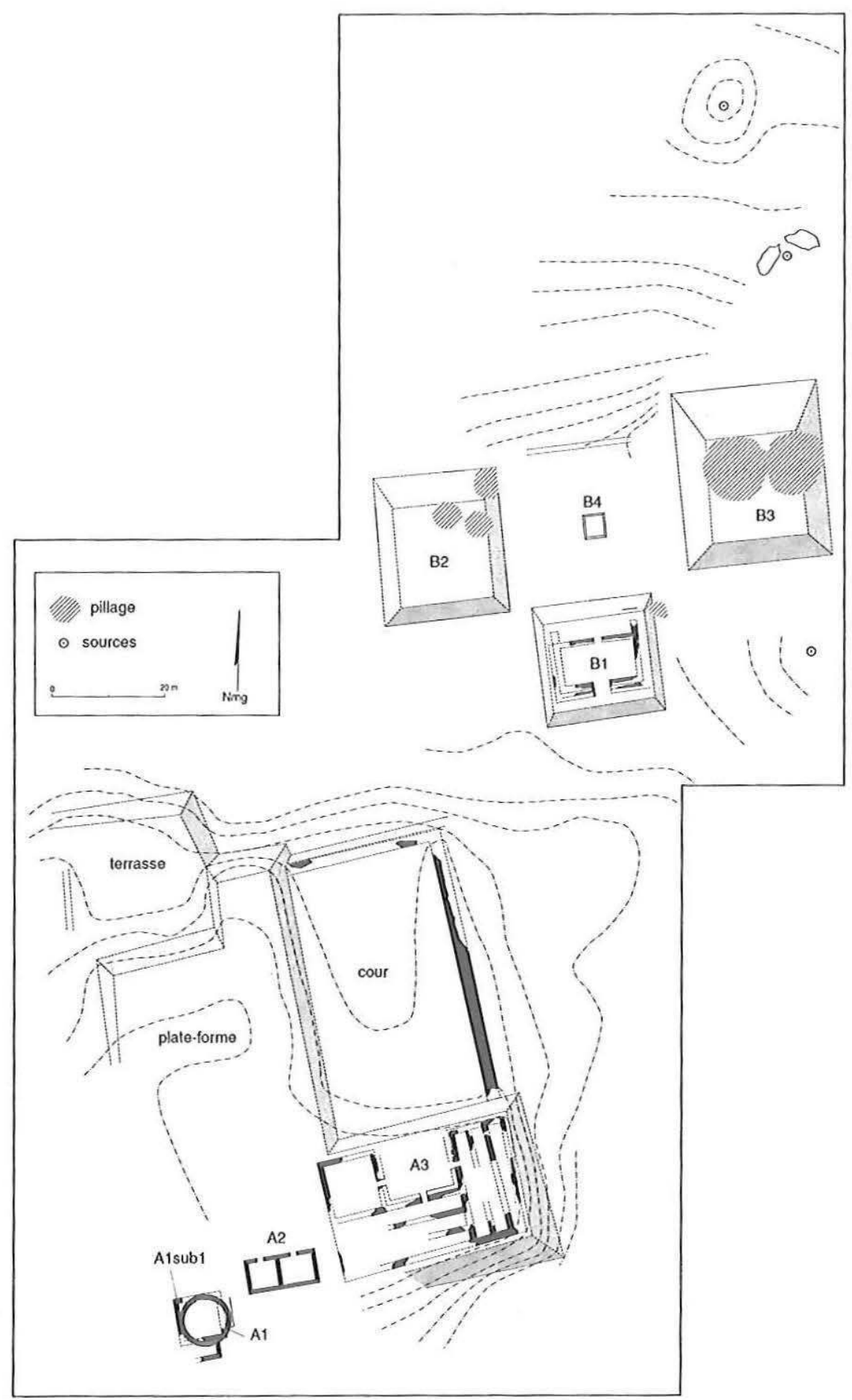

Fig. 3 - Relevé topographique des groupes A et B de Nogales (relevé D. Michelet) 
Le seul édifice pour lequel nous disposons d'un plan relativement complet est la structure A2-sub. Il s'agit d'un bâtiment de plan quadrangulaire de $14 \mathrm{~m}$ sur $12 \mathrm{~m}$ — associé à deux petites pièces annexes — pourvu d'une entrée sur au moins trois de ses côtés et dont l'espace interne ne semble comporter aucun cloisonnement. La fonction de cet édifice reste énigmatique en l'absence d'aménagements intérieurs significatifs ou de concentrations de matériel permettant de déduire des aires d'activité. Tout au plus, ses dimensions et son plan suggèrent une fonction publique ou communautaire. La présence d'une étape de réaménagement intérieur (construction d'une banquette contre le mur nord) a permis de distinguer une sous-phase récente (A2-sub1) d'une sous-phase ancienne (A2-sub2). Cette distinction est également perceptible dans l'évolution des types céramiques : en Nogales ancien, les types sont proches de ceux que nous avons signalés pour l'occupation initiale alors qu'en Nogales récent on perçoit une influence du centre-nord du Michoacan à travers des récipients qui évoquent les types Ciénega rouge, Ciénega rouge négatif et Lupe incisé définis dans la région de Zacapu (Michelet \& al. 1989 ; Michelet 1993 ; Pereira 1999). L'assemblage céramique de cette époque constitue en quelque sorte un intermédiaire entre les traditions du Bajio guanajuatense et celles du centre-nord du Michoacan.

À l'échelle du massif, et dans l'état actuel de nos connaissances, l'implantation de la phase Nogales paraît limitée au Groupe A (et peut-être B) de Nogales et à certains secteurs du site voisin d'El Moro.

La phase Barajas (de 750 à 950 apr. J.-C.)

Cette nouvelle phase constitue, à bien des égards, une rupture notable avec l'occupation antérieure. Elle est d'abord marquée par une colonisation rapide et massive des versants du massif du Barajas. C'est de cette période que sont datés la plupart des sites repérés et l'essentiel des structures visibles en surface. Elle se caractérise, en outre, par des réalisations monumentales complexes dont Nogales fournit les exemples les plus spectaculaires. Cette architecture se signale par l'emploi de dalles désormais plus petites et dont la tranche destinée au parement des murs est soigneusement régularisée par des enlèvements. Dans le Groupe A, l'édification des structures de cette époque semble précédée par un épisode de destruction des constructions antérieures (traces d'incendie dans certains cas). Le groupe acquiert alors son aspect définitif et ne subira que de faibles modifications ultérieures. Les trois structures fouillées à ce jour (A1, A2 et A3) se trouvent dans la partie sud d'une vaste plate-forme en $\mathrm{L}$ présentant des avancées terrassées dans son extrême nord-ouest (Figure 3). Cette plate-forme détermine, par ailleurs, les limites sud et ouest d'un grand patio rectangulaire de $47 \mathrm{~m}$ de long sur $26 \mathrm{~m}$ de large dont les deux autres côtés sont marqués par d'épais murs de dalles (2,50 $\mathrm{m}$ de large). Au sud de ce patio, on accédait à la structure A3 par l'intermédiaire d'un escalier. A3 est l'édifice le plus imposant du groupe (34 m de long sur 21 m de large) : c'est un bâtiment en forme de $U$ : la cour autour de laquelle il s'organise, compte des banquettes adossées aux murs périphériques et est ouverte au nord. L'accès aux espaces intérieurs se faisait par l'intermédiaire de trois portes aménagées sur les côtés est, ouest et sud. Les pièces n'ont pas été dégagées mais plusieurs éléments visibles en surface permettent d'en comprendre l'organisation générale. À l'ouest, on entrait dans une grande pièce carrée d'une dizaine de mètres de côté qui semble dépourvue de divisions internes. En revanche, à l'est et au sud, on accédait à une série de pièces étroites et longues disposées parallèlement. 
La structure A2 se trouve à l'ouest de la précédente. Elle se présentait en surface sous la forme d'un épandage de dalles. La fouille a mis au jour les murs d'une construction rectangulaire de 11,50 $\mathrm{m}$ de long sur 4,40 $\mathrm{m}$ de large (Figure 4) pourvue de deux portes ouvrant vers le nord. À l'intérieur, des restes correspondant à la base d'une cloison ont été retrouvés. Enfin, la structure A1, datée de cette phase, n'a pu être intégralement fouillée car elle se trouvait recouverte par une structure ronde plus tardive (A1-sup). Les indices disponibles montrent qu'elle était formée d'une pièce carrée d'environ $7 \mathrm{~m}$ de côté dont l'accès n'a pas pu être localisé. À l'est, une petite terrasse lui était accolée tandis qu'au sud nous avons partiellement dégagé les murs d'une pièce annexe de 2,50 $\mathrm{m}$ de large.

En ce qui concerne la céramique, la phase Barajas voit la quasi-disparition de certains types de la phase antérieure (comme le Blanco levantado) et l'apparition de types nouveaux tels que les jarres orange à décor peint en noir, les jarres rouges à décor gravé après cuisson, les jarres orange à surface externe polie et décorée de bandes rouges et de motifs au négatif, les récipients brun rougeâtre grossiers. D'autres types déjà présents - tels que les récipients Rouge sur bai, Brum fin avec ou sans incisions - subsistent mais présentent des formes ou des décors nouveaux. Globalement, la céramique de cette phase montre plus de similitudes avec la région correspondant au nord-ouest du Guanajuato, zone de La Gloria en particulier; ( $c f$. Sánchez Correa 1995) et aux Altos de Jalisco. Des liens avec le nord du Michoacan sont également perceptibles.

Le début du Postclassique (de 950 à 1100 apr. J.-C.)

Après l'abandon sans doute rapide de la plupart des sites habités en phase Barajas, une occupation limitée et probablement assez brève, est attestée dans le Groupe A de Nogales. La seule construction que nous ayons pu identifier pour cette période est une structure de plan circulaire (A1-sup ; Figure 5) construite au-dessus de A1. L'occupation est également représentée par des dépotoirs dont on trouve les traces sur les ruines des constructions antérieures, certaines ont peut-être été «squattées ». Du point de vue de la culture matérielle, cette phase se caractérise par une industrie lithique sensiblement plus abondante qu'auparavant et par une céramique plus simple et plus grossière. Les types brun et rouge fin monochromes perdurent mais présentent des formes et des décors sensiblement différents. Notons en outre la présence de tessons de céramique fine rouge incisée, parfois associés à du pigment blanc, imitant des types connus au Jalisco et au Queretaro ${ }^{5}$ à la même période ou provenant directement de ces régions. Un vase tripode anthropomorphe a également été mis au jour dans les niveaux correspondant à cette période.

Le Postclassique récent (1400-1521 apr. J.-C.)

L'existence d'une occupation tardive n'a été repérée que dans les couches superficielles de la place du Groupe B de Nogales. La présence d'un foyer en cuvette associé à un abondant matériel lithique (essentiellement des pointes de projectiles en obsidienne) et osseux (faune) laisse penser qu'il s'agit là des vestiges d'un campement ou d'une halte de chasseurs. La présence de pointes de flèches miniatures de forme triangulaire à base concave ainsi que de deux fragments d'objets de céramique tarasque (fragment de pipe et de bec verseur) montre clairement qu'il s'agit d'une occupation tardive. 


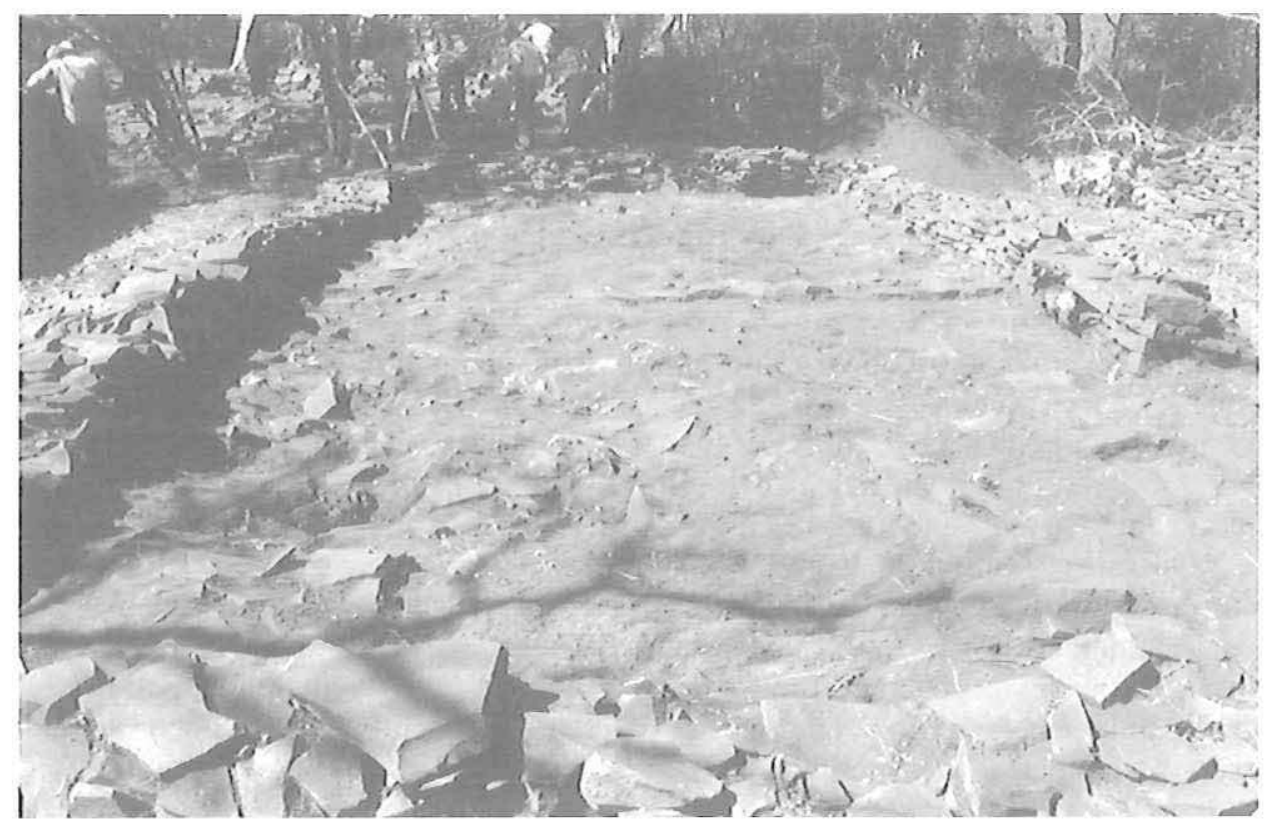

FIG. 4 - Vue générale de la structure A2 depuis l'est (photo G. Migeon)

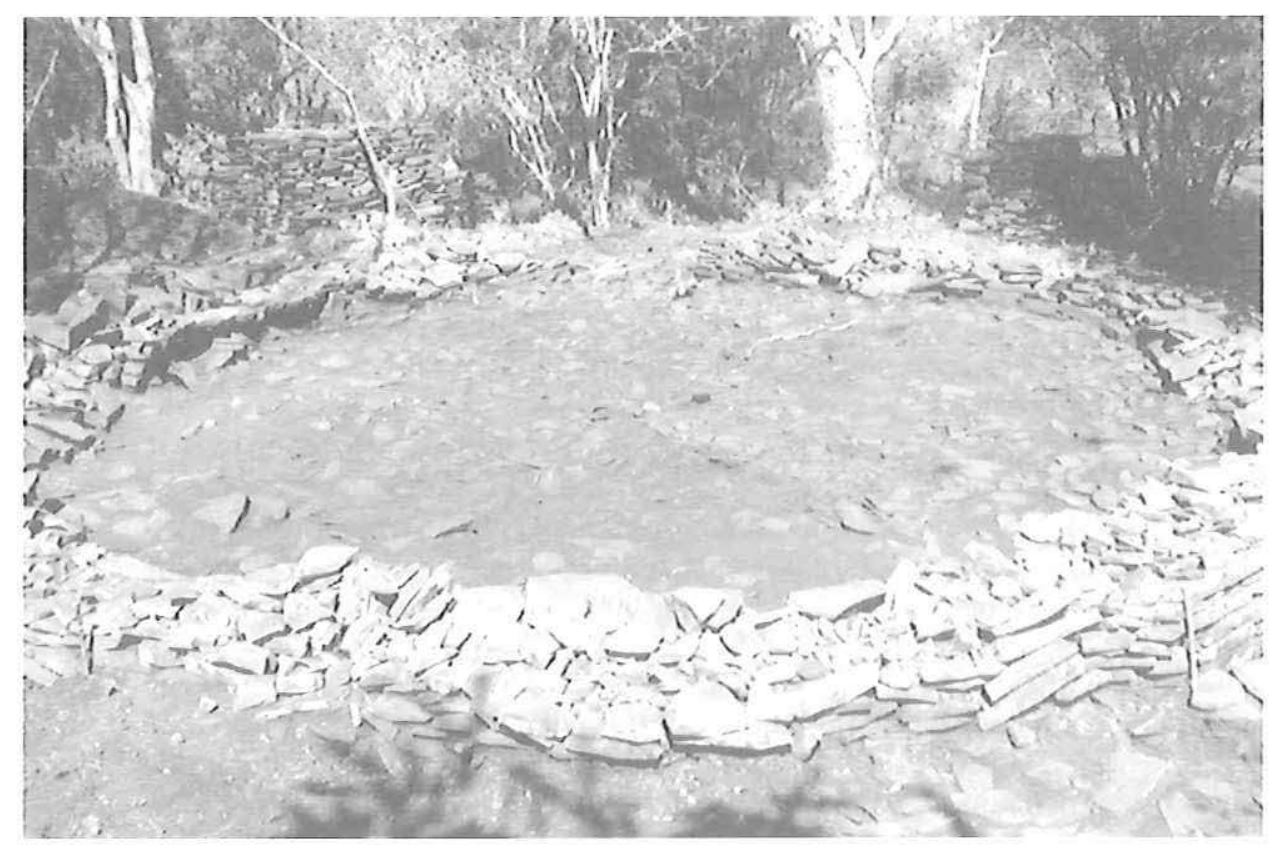

FIG. 5 - Vue générale de la structure Al-sup depuis le sud (photo G. Pereira) 


\section{L'organisation des sites de la phase Barajas}

Comme nous l'avons déjà dit, la plupart des sites et des structures répertoriées en surface datent donc très probablement de la phase Barajas qui marque l'apogée démographique de la zone. Les réalisations architecturales de cette période sont encore bien préservées, ce qui permet d'entrevoir la structuration des établissements et quelques-unes de ses possibles implications socio-politiques.

Les sites les plus importants constituent un ensemble continu qui couvre une grande partie du versant nord du massif du Barajas (Figure 2). On constate des interruptions dans l'occupation qui ont été prises pour limites entre les sites, mais celles-ci correspondent en fait aux ravins, plus ou moins profonds, qui entaillent perpendiculairement le versant. Ces limites étant généralement imposées par la topographie, il n'est pas interdit de penser qu'une majorité des établissements a pu former jadis en réalité une seule et même entité. À l'échelle de chaque établissement distingué, les structures les plus importantes se situent généralement dans la partie la plus haute, sur des emplacements élevés naturellement ou artificiellement qui offrent une vue dégagée sur la vallée. La taille et la complexité des structures décroît généralement vers l'aval, les parties basses étant souvent occupées par des terrasses d'habitat modeste. Ce type d'habitat est particulièrement bien représenté sur le site d'El Moro où un réseau relativement dense de petites terrasses (une terrasse tous les 10 m environ) s'étend sur près de 100 ha. On trouve des secteurs comparables dans les parties basses des sites de Camposanto et Yacata El Angel. Le site de Nogales se distingue, quant à lui, de la plupart des autres sites : limité à l'est et à l'ouest par de profonds ravins, il compte presque exclusivement des structures monumentales de grandes dimensions. Ce caractère pourrait s'expliquer par le fait que Nogales aurait occupé localement une position privilégiée et fonctionné peut-être comme le centre politique et religieux de la zone. Un autre site attire l'attention : Los Toriles. Il s'agit là d'un vaste éperon naturel barré au sud par une impressionnante muraille de pierre (Figures 2 et 6 ) de près de $600 \mathrm{~m}$ de long ${ }^{6}$; ce site, clairement défensif, est localisé au cœur du massif, juste en amont de Nogales. Étant donné sa situation et l'absence de vestiges d'habitat à l'intérieur de son périmètre, il est vraisemblable qu'il a été conçu pour servir de zone de refuge en cas de conflit. L'investissement engagé par les habitants du massif pour la réalisation de la muraille témoigne sans aucun doute, quant à lui, d'une situation politique tendue. Par ailleurs, il est intéressant de signaler que des zones de concentration de petites structures souterraines, sans doute liées au stockage, ont été repérées dans plusieurs sites. Des ensembles de ce type ont été observés et partiellement fouillés sur les sites d'El Moro et de Casas Tapadas. Dans ce dernier établissement, ces aménagements formaient même les sous-sols de certains bâtiments, ce qui constitue un élément particulièrement original.

Si l'on s'intéresse à présent à l'organisation des ensembles architecturaux enregistrés lors des travaux de prospection et de topographie, on remarque, au premier abord, une grande diversité des réalisations. L'analyse de près de 140 structures bâties et aménagements annexes (cours, places) permet cependant d'esquisser une typologie et d'observer un certain nombre de régularités significatives. Au sein de la grande variété de constructions observées, au moins deux types de complexes définis par des associations d'éléments récurrents ont pu être identifiés. 


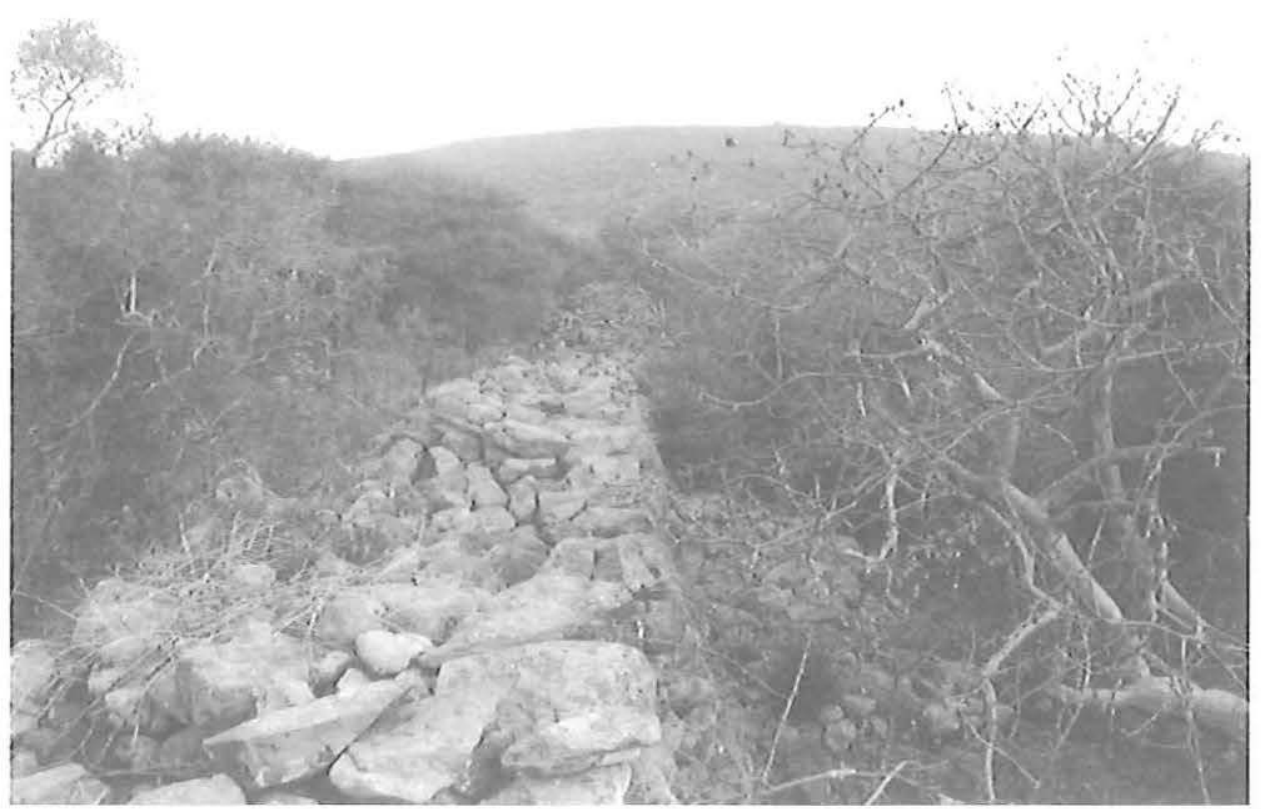

FIG. 6 - Vue de la muraille de Los Toriles depuis l'est (photo G. Migeon)

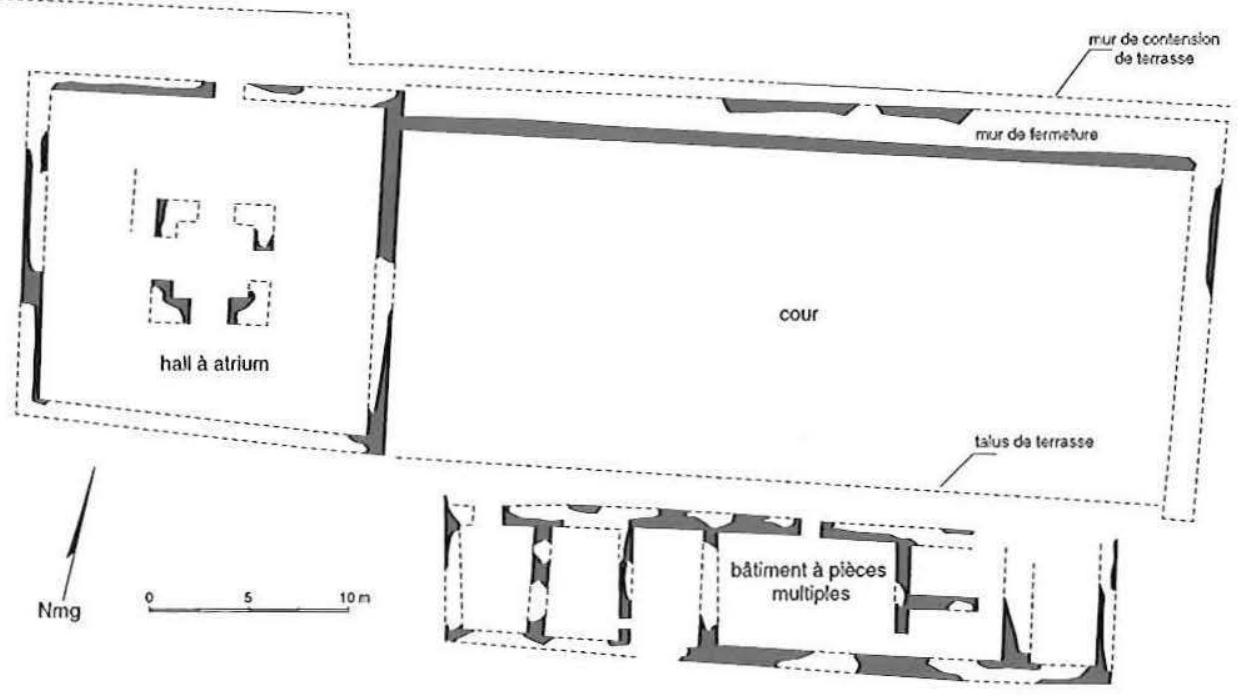

Fig. 7 - Le Groupe A de Camposanto (relevé D. Michelet). Un exemple de complexe de type A 
Le premier type (dit " Complexe A ", voir Figure 7) se compose d'un bâtiment à pièces multiples dont la disposition est variable - plan allongé (Groupe A de Camposanto), en L (Groupe A de Nogales ${ }^{7}$ ) ou en bloc (Groupe C de Nogales, Groupes $\mathrm{C}$ et $\mathrm{H}$ de Yacata El Angel) - auquel est associé un édifice de plan carré ou sub-carré de grandes dimensions (10 à $16 \mathrm{~m}$ de côté dans la plupart des $\operatorname{cas}^{8}$ ) qui peut être interprété comme un hall pourvu d'un atrium central : c'est en tout cas ce que laisse supposer la présence de piliers délimitant un espace central quadrangulaire dans le Groupe A de Camposanto et le Groupe C de Yácata El Angel ; dans ce dernier cas, c'est la fouille qui a permis d'identifier ce type d'aménagement et d'envisager sa présence dans d'autres constructions du même type. Notons que le hall peut être totalement indépendant du bâtiment à pièces multiples (Groupe $\mathrm{C}$ de Nogales, A de Camposanto) ou encore être accolé à ce dernier (Groupe $\mathrm{A}$ de Nogales, $\mathrm{C}$ et $\mathrm{H}$ de Yacata El Angel). Mais il semble constituer, en tout état de cause, un espace distinct auquel on accédait depuis l'extérieur par une porte unique. Un troisième élément est souvent associé à ces deux catégories d'édifices. Il s'agit d'une cour rectangulaire (Groupe A de Nogales, A de Camposanto, $\mathrm{H}$ de Yacata El Angel), parfois sub-carrée (Groupe C de Nogales) qui se trouve en contrebas des plates-formes sur lesquelles sont édifiés les bâtiments que nous venons de décrire. Elle est, par ailleurs, fermée par d'épais murs de pierre qui en interdisent ou limitent l'accès depuis l'extérieur.

Le second type d'association (« Complexe B », voir structures B1, B2, B3 et B4 de la Figure 3) est constitué d'une à trois bases pyramidales — ou plates-formes hautes - qui s'organisent autour d'une place. Dans plusieurs cas, nous avons pu identifier l'existence d'une superstructure construite au sommet de la base pyramidale. Il s'agit d'un édifice rectangulaire de 8 à $13 \mathrm{~m}$ de long formé d'une pièce unique et dont la façade devait être pourvue, dans plusieurs cas (structures B1 et D1 de Nogales, D1 et D3 de Yacata El Angel), d'un portique délimité sur les côtés par l'avancée des murs latéraux. La place elle-même, de forme carrée ou subcarrée, était parfois fermée par un mur (Groupes B et D de Nogales, A d'El Moro). Dans plusieurs cas, les vestiges d'un petit autel ont été identifiés en son centre (structure B4 de Nogales, A4 d'El Moro, A5 de Camposanto et A2 et G2 (?) de Yácata El Angel). La fouille de l'autel B4 de Nogales nous a apporté quelques précisions concernant ce type de structure : c'est une simple plate-forme basse de plan carré $(4,10 \mathrm{~m}$ sur $3,90 \mathrm{~m})$ aux parements en talus.

Il convient à présent de s'interroger sur la signification de ces deux types de complexes qui réunissent les réalisations les plus monumentales du massif du Barajas. Il est d'abord intéressant de remarquer que les deux catégories que nous venons de distinguer se trouvent systématiquement associées dans un même site (l'espace qui les sépare est toujours inférieur à $50 \mathrm{~m}$ ), ce qui laisse présager une certaine complémentarité fonctionnelle. En outre, les régularités constatées quant aux éléments constitutifs et à leur agencement spatial montrent clairement que cette architecture obéit à des schémas normatifs.

La présence de ces complexes, associés à Nogales comme sur des sites voisins tels que El Moro, Camposanto, Yacata El Angel et peut-être La Perra, évoque clairement 
une organisation socio-politique cohérente. Il existe toutefois des différences notables entre ces sites en ce qui concerne les dimensions. Les groupes du haut de Nogales (A, $\mathrm{B}, \mathrm{C}, \mathrm{D}$ ) sortent très nettement du lot par leur monumentalité (la surface du Groupe C dépasse les $6000 \mathrm{~m}^{2}$ ). Suivent en importance les groupes du bas de Nogales (Groupe G) et d'El Moro (Groupe A), puis les complexes plus modestes situés sur les autres sites (moins de $500 \mathrm{~m}^{2}$ ).

Concernant les complexes du type $\mathrm{B}$, on ne peut que constater leur adéquation avec un schéma mésoaméricain bien connu (temple-pyramide/place/autel) dont le caractère religieux ne fait aucun doute. En revanche, l'interprétation des complexes du type A est plus délicate étant donné que ces ensembles associent des éléments moins répandus. Il est d'ailleurs vraisemblable qu'ils aient abrité plusieurs types d'activités. Les bâtiments à pièces multiples permettent de supposer une fonction résidentielle. Leurs dimensions et leur complexité indiquent néanmoins qu'il ne s'agit pas là d'habitations communes ${ }^{9}$. Par ailleurs, la présence de nombreuses pièces exiguës à l'intérieur de ces structures permet de penser qu'une partie de celles-ci était vouée au stockage. Les halls à atrium et les grandes cours fermées évoquent quant à eux une vocation publique (espaces de rassemblement et de réunion). Ces éléments présentent apparemment peu de points communs avec la plupart des sites du Bajio qui s'insèrent, durant l'époque classique, dans la tradition des Patios Hundidos ${ }^{10}$. En revanche, par certains aspects, les sites du Barajas évoquent les cultures du nord-ouest de la Mésoamérique. C'est en particulier le cas des halls à atrium dont on trouve des équivalents dans des sites de la culture Chalchihuites tels que La Quemada et Altavista (Zacatecas) ${ }^{11}$. Ce type d'édifice, qui constitue apparemment un élément caractéristique de cette culture, a été interprété comme un espace lié à des cérémonies collectives à connotation guerrière (Hers 1995). Rappelons qu'il sera introduit dans le Mexique central au Postclassique ancien pour s'intégrer à la culture toltèque laquelle aurait contribué à sa diffusion vers Chichen Itza. Dans cette perspective, on peut penser que les sites du Barajas constituent un intermédiaire au même titre que San Antonio Carupo (Faugère-Kalfon 1991).

\section{CONCLUSION}

Les premières recherches menées sur le site de Nogales et ses environs nous ont permis de brosser un tableau général concernant l'occupation de ce secteur de la vallée du Lerma. Les résultats obtenus confirment tout d'abord nos hypothèses concernant la position chronologique de l'occupation majeure du massif du Barajas qui se situe entre 750 et 950 de notre ère, c'est-à-dire à l'Épiclassique. Ils montrent par ailleurs que cette occupation de la phase Barajas a été précédée puis suivie par des occupations bien identifiables quoique modestes. Dans ce contexte, l'importance et la relative brièveté de l'occupation Barajas soulèvent de nombreuses questions. L'expansion démographique qu'elle représente ne semble pas pouvoir s'expliquer par un accroissement naturel des groupes présents antérieurement ; aussi est-il tentant d'envisager un phénomène migratoire aboutissant à la colonisation du massif peu peuplé jusque-là. Ce phénomène migratoire pourrait d'ailleurs être le fait des habitants vivant dans la plaine alentour; sous forme de regroupement et/ou de relocalisation de l'habitat. Il pourrait correspondre aussi à des mouvements de plus grande amplitude 
(comme pourrait le faire supposer l'apparition de types céramiques rappelant la région des Altos de Jalisco). Ces deux hypothèses ne sont d'ailleurs pas incompatibles.

Les travaux qui seront développés lors des années à venir auront pour objectif d'affiner notre perception des phénomènes historiques qui marquent le Barajas à la fin du premier millénaire de notre ère. Il faudra tenter de comprendre en particulier la nature des changements qui interviennent en phase Barajas ainsi que les raisons qui ont entraîné l'abandon apparemment rapide ${ }^{12}$ des grands établissements de cette époque. Il faudra pour cela compléter notre connaissance des sites et approfondir certains thèmes tels que la fonction des structures repérées ou la nature et l'évolution des pratiques funéraires et des populations inhumées. En outre, il faudra amorcer une recherche concernant les conditions environnementales dans lesquelles ces groupes préhispaniques ont vécu afin de vérifier dans quelle mesure celles-ci ont pu influer sur l'histoire de la région.

\section{ANNEXE}

\begin{tabular}{|c|c|c|c|c|}
\hline $\begin{array}{l}\text { RÉFÉ- } \\
\text { RENCES }\end{array}$ & PROVENANCES-PHASES & ÂGES & DATES CALIBRÉES 2 & COMMENTAIRES \\
\hline Gif-11494 & $\begin{array}{l}\text { Nogales, Groupe A, (UE } 612 \text { ) } \\
\text { str. A1, remblai « recouvrant » } \\
\text { la str. } \\
\text { Postclassique ancien }\end{array}$ & $1570 \pm 50 \mathrm{BP}$ & $\begin{array}{l}\text { AD } 393(442,448, \\
468,482,530) 616\end{array}$ & $\begin{array}{l}\text { dates trop ancien- } \\
\text { nes (le remblai en } \\
\text { question a dû re- } \\
\text { prendre du charbon } \\
\text { plus ancien) }\end{array}$ \\
\hline Gif-11495 & $\begin{array}{l}\text { Nogales, Groupe A, (UE 622) } \\
\text { str. A1, sols } 2 \\
\text { BARAJAS }\end{array}$ & $1140 \pm 30 \mathrm{BP}$ & $\begin{array}{l}\text { AD 782 }(894,925 \text {, } \\
\text { 935) } 983\end{array}$ & $\begin{array}{l}\text { toutes les dates sont } \\
\text { possibles }\end{array}$ \\
\hline Gif-11498 & $\begin{array}{l}\text { Nogales, Groupe A, (UE 713) } \\
\text { str. A2 } \\
\text { sol supérieur } \\
\text { BARAJAS }\end{array}$ & $1605 \pm 30 \mathrm{BP}$ & AD 396 (429) 539 & $\begin{array}{l}\text { dates trop ancien- } \\
\text { nes (reprise de char- } \\
\text { bons anciens?) }\end{array}$ \\
\hline Gif-11496 & $\begin{array}{l}\text { Nogales, Groupe A, (UE 711) } \\
\text { couche de destruction de la } \\
\text { str. A2subl } \\
\text { Début BARAJAS }\end{array}$ & $1300 \pm 50 \mathrm{BP}$ & AD $649(689) 862$ & $\begin{array}{l}\text { postérieur à } \\
\text { Gif-11497 }\end{array}$ \\
\hline Gif-11369 & $\begin{array}{l}\text { Nogales, Groupe A, (UE 124) } \\
\text { sondage 3, } \\
\text { soubassement du sol } 2 \\
\text { Début BARAJAS }\end{array}$ & $1340 \pm 40 \mathrm{BP}$ & AD 644 (671) 776 & $\begin{array}{l}\text { postérieur à } \\
\text { Gif-11370 }\end{array}$ \\
\hline Gif- 11370 & $\begin{array}{l}\text { Nogales, Groupe A, (UE 126) } \\
\text { sondage 3, remblai sous sol } 3 \\
\text { NoGALES récent }\end{array}$ & $1225 \pm 40 \mathrm{BP}$ & AD 685 (788) 893 & $\begin{array}{l}\text { antérieur à } \\
\text { Gif-11369 }\end{array}$ \\
\hline Gif-11497 & $\begin{array}{l}\text { Nogales, Groupe A, (UE 712) } \\
\text { str. A2subl } \\
\text { sol supérieur } \\
\text { NoGALES récent }\end{array}$ & $1590 \pm 40 \mathrm{BP}$ & AD 393 (433) 595 & $\begin{array}{l}\text { antérieur à } \\
\text { Gif-11496 }\end{array}$ \\
\hline
\end{tabular}

Dates ${ }^{14} \mathrm{C}$ de NOGALES (saisons 1998 et 1999)

(Dans la colonne Dates calibrées sont figurées en gras les dates vers lesquelles il faudrait, selon les indices stratigraphiques, situer les échantillons analysés). 


\section{NOTES}

1. Ce programme est mené par différents membres de l'ArchAm - Archéologie des Amériques (UMR 8096 CNRS-Université Paris I) grâce à des financements du ministère des Affaires étrangères et du CNRS. Rappelons que d'autres thématiques de recherche sont développées au sein du même projet. Celles-ci portent sur la culture préclassique de Chupicuaro (fouilles dans la région de Puruagüita, $c f$. Darras \& al., 1999) et une de ses évolutions régionales (fouilles de Loma Alta, $c f$. Carot \& al., 1998). Concernant les travaux de terrain sur le massif du Barajas, outre les trois auteurs de cet article, ils ont bénéficié de la participation de Laura Almendros, Rosalba Berumen, Séverine Bortot, Jean-Pierre Courau, Ninon Roose et Patrice Wuscher.

2. D’après la définition proposée par le Diccionario Porría de historia, biografia y geografia $\left(5^{\mathrm{e}}\right.$ édition, 1986), le Bajio correspond à la partie de l'altiplano mexicain située au nord de l'axe néo-volcanique et au sud de la Sierra de Guanajuato. Il s'agit d'un bassin alluvial qui est principalement drainé par le Río Lerma. Ce dernier coule d'est en ouest dans une vallée qui, depuis Celaya-Acámbaro, à l'est, jusqu'à La Piedad, à l'ouest, s'échelonne en paliers successifs. Les fonds de vallées sont très fertiles et sont constitués de sols épais formés de colluvions d'origine volcanique et de dépôts alluviaux et lacustres.

3. À ce sujet, on mentionnera en particulier les recherches réalisées dans le cadre du projet Michoacan (Michelet et al., 1989 ; Faugère-Kalfon, 1996), au sud, et dans celui du sauvetage de La Gavia/La Purisima, au nord (Taladoire \& Rodriguez Loubet, 1979; Taladoire, 1993 ; Sánchez Correa, 1995), de même que les fouilles en cours réalisées sur le site voisin de Plazuelas (Castañeda, 2000 ; Juárez Cossío, 1999).

4. Un ensemble de 7 dates ${ }^{14} \mathrm{C}$ a été obtenu à ce jour (voir tableau annexe). Celles-ci ont été effectuées sous la responsabilité de Michel Fontugne au sein du Laboratoire des sciences du climat et de l'environnement de Gif-sur-Yvette.

5. Ils présentent en particulier certaines similitudes avec le type Xajay de San Juan del Río (J.-C. Saint-Charles, com. pers. 2000) et le type Cojumatlan polychrome du complexe Aztatlan (S. Ramírez U., com. pers. 2001).

6. Cette localisation de la muraille, qui protège l'accès du site depuis le sud, est a priori surprenante. Cette direction (le sud) correspond en effet au sommet du massif et l'on imagine par ailleurs que les habitants du Barajas cherchaient plutôt à se prémunir de possibles incursions depuis le nord. En réalité, la topographie des lieux explique tout à fait cette apparente contradiction. En effet, le site est naturellement protégé dans sa partie nord par des ravins abrupts ce qui n'est pas le cas au sud où le terrain est presque plan. Cet ouvrage avait donc pour objectif de défendre la place dans le cas où les assaillants auraient cherché à la prendre à revers.

7. Nous nous référons ici à la seule structure A3. Comme nous l'avons mentionné plus haut, l'ensemble de cette construction forme un U. Dans le cadre de l'analyse proposée ici, nous sommes conduits à distinguer deux espaces fonctionnels distincts : l'espace à pièces multiples est formé par l'aile est et la partie centrale (sud) de l'édifice tandis que l'aile ouest est formée d'une grande salle que nous interprétons comme un hall.

8. La structure $\mathrm{Cl}$ du Groupe $\mathrm{C}$ de Nogales, avec ses $36 \mathrm{~m}$ de côté, est exceptionnelle et se distingue en particulier par sa monumentalité.

9. Les habitats ordinaires correspondent aux maisons à une ou deux pièces construites en pierre ou, plus souvent sans doute, en matériaux périssables. Elles sont généralement édifiées sur une petite terrasse.

10. À cet égard, notre opinion diverge du point de vue de Cardenas (1999, p. 190) qui inclut Nogales dans cette tradition architecturale. En effet, ce dernier croit voir dans le Groupe C de Nogales deux patios hundidos là où il s'agit, d'un côté, de la place centrale du groupe (qui n'est délimitée par des plates-formes que sur trois côtés), de l'autre d'une dépression qui sert d'atrium à la structure $\mathrm{Cl}$.

11. D'autres halls à colonnes ou piliers délimitant des atriums centraux ont été aussi signalés dans des sites comme La Tirisia ou El Teúl, au sud de La Quemada, mais aussi au Cerro de Las Vívoras et au Cerro Moctehuma, près d'Alta Vista ( $c f$. Hers, 1995, p. 102). Néanmoins, l'absence de plan publié de ces édifices limite fortement les comparaisons. Remarquons que les halls du Barajas semblent dépourvus de portique en façade à la différence des exemples septentrionaux et de San Antonio Carupo, pourtant situé plus au sud.

12. Il est intéressant de remarquer que certains indices évoquent un abandon concerté des lieux. C'est ce que montrent divers bâtiments dont les accès ont été soigneusement murés de façon à les rendre inutilisables. Ces dispositifs de condamnation ont été observés dans diverses structures du Groupe D de Nogales et dans le hall à atrium du Groupe C de Yacata El Angel. Dans ce dernier cas, la fouille a montré que les espaces situés entre chaque pilier avaient été bouchés. D’autres structures repérées en surface et qui semblent dépourvues d'accès pourraient illustrer ce même phénomène. À l'avenir, il sera nécessaire de vérifier systématiquement cet aspect. 


\section{RÉFÉRENCES BIBLIOGRAPHIQUES}

Armillas, P., 1964 - « Condiciones, ambiente y movimientos de pueblos en la frontera norte de Mesoamérica. », in : Homenage a Fernando Márquez Miranda, pp. 62-82, Publicaciones del Seminario de Estudios Americanistas y el Seminario de Antropología Americana, Universidades de Madrid y Sevilla, Madrid.

—, 1969 - "The arid frontier of Mexican civilization. ", in : Transactions of the New York Academy of Sciences, Série II, vol. 31, nº 6, pp. 6-8, New York.

BranifF, B., 1989 - «Oscilación de la frontera norte de Mesoamérica : un nuevo ensayo ", Arqueología, 1, pp. 99-114.

—, 1994 - «La frontera septentrional de Mesoamérica. », in : Historia Antigua de México, vol. I, L. Manzanilla \& L. López Luján (coord.), pp. 113-143, INAH-UNAM-Porrúa, México.

—, 1999 - Morales, Guanajuato, y la tradición tolteca, INAH, Colección científica nº 395, México.

Cárdenas, E., 1999 - El Bajio en el Clásico, El Colegio de Michoacán, Zamora.

Carot, P., M.-F. Fauvet-Berthelot, L. Barba, K. Link, A. Ortíz \& A. Hesse, 1998 - « La arquitectura de Loma Alta, Zacapu, Michoacán ", in : IV Coloquio de Occidentalistas : arqueología, historia y medio ambiente, pp.345-351, Universidad de GuadalajaraORSTOM, Guadalajara.

Castañeda, C., 2000 - « Las maquetas de Plazuelas, Guanajuato », Arqueologíamexicana, 46, pp. 76-79.

Castañeda C., L. Flores, C. Contreras, A.-M. Crespo \& J.-C. Saint-Charles, 1988 «Interpretación de la historia del acentamiento en Guanajuato », in : Primera reunión sobre las sociedades prehispánicas en el centro-occidente de México, Cuaderno de trabajo $n^{\circ} 1$, pp. 321-355, INAH, Querétaro.

Darras, V., B. Faugère-Kalfon, C. Durlet, C. Liot, J. Reveles, R. Bérumen, O. Cervantés, C. Caillaud \& C. David, 1999 - « Nouvelles recherches sur la culture de Chupicuaro (Guanajuato, Mexique) », Journal de la Société des Américanistes, 85, pp. 343-351.

Faugère-Kalfon, B., 1991 - «San Antonio Carupo (centro-norte de Michoacán, México) : nuevas evidencias de ciertas transformaciones en el inicio del Postclásico », Journal de la Société des Américanistes, 77, pp. 45-61.

—, 1996 - Entre Zacapu y Río Lerma : culturas en una zona fronteriza, Cuadernos de Estudios Michoacanos, $n^{\circ}$ 7, CEMCA, México.

Hers, M.-A., 1995 - «Las salas de columnas en La Quemada », in : Arqueología del norte y occidente de México. Homenaje al Doctor J. Charles Kelley. B. Dahlgren \& D. Soto de Arechavaleta (ed.), pp. 93-113, UNAM, México.

JuÁrez Cossío, D., 1999 - «Exploraciones en San Juan el Alto, Municipio de Pénjamo, Guanajuato », Arqueologia, 22, pp. 41-68.

Michelet, D., 1984 - Río Verde, San Luis Potosi (Mexique), Collection Études Mésoaméricaines, I-9, CEMCA, México.

—, 1993 — « La cerámica de las Lomas en la secuencia cerámica regional », in : Arqueología de las Lomas en la Cuenca lacustre de Zacapu, Michoacán, México, Cuadernos de Estudios Michoacanos n 5, pp. 149-155, CEMCA, México. 
Michelet, D., M.-C. Arnauld, \& M.-F. Fauvet-Berthelot, I989- «El proyecto del CEMCA en Michoacán. Etapa I : un balance », Trace, 16, pp. 70-87, CEMCA, México.

PEREIRA, G., 1999 - Potrero de Guadalupe : anthropologie funéraire d'une communauté prétarasque du nord du Michoacan, Mexique, BAR International Series 816, Oxford.

Rodriguez Loubet, F., 1985 - Les Chichimèques, Collection Études Mésoaméricaines, I-12, CEMCA, México.

SÁNChez Correa, S., 1993 - « Comentarios sobre algunos sitios arqueológicos localizados en el suroeste de Guanajuato ", Cuadernos de arquitectura mesoamericana, 25, pp. 51-57.

—, 1995 - La Gavia, Guanajuato : aproximación al desarrollo cultural de una porción del Bajio noroccidental, tesis de licenciatura, ENAH, México.

Stresser-PÉAn, G., 1977 - Sam Antonio Nogalar, Collection Études Mesoaméricaines, 3, MAEFM, México.

Taladoire, E., 1993 - La Gavia et la Purisima. Archéologie de sauvetage dans l'État de Guanajuato (1977), Document de travail nº 8, Centre de Recherches en Archéologie Précolombienne, université de Paris I, Paris.

Taladoire, E. \& F. Rodriguez Loubet, 1979 - « Fouilles de sauvetage dans l'État du Guanajuato, Mexique », Journal de la Société des Américanistes, 66, pp. 295-303.

Zepeda, G., 1988 - « Nogales : fortaleza tarasca en el estado de Guanajuato », in : Primera reunión sobre las sociedades prehispánicas en el centro-occidente de México, Cuaderno de trabajo n 1, pp. 299-306, INAH, Querétaro. 